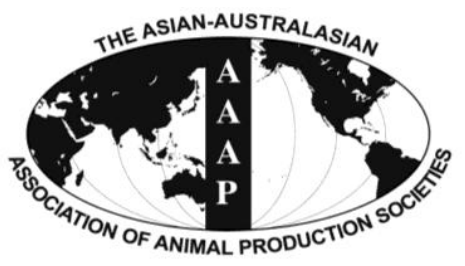

Asian-Aust. J. Anim. Sci.

Vol. 25, No. $10: 1381$ - 1388

October 2012

www.ajas.info

http://dx.doi.org/10.5713/ajas.2012.12240

\title{
Effects of Defaunation on Fermentation Characteristics and Methane Production by Rumen Microbes In vitro When Incubated with Starchy Feed Sources
}

\author{
W. Z. Qin ${ }^{1,2}$, C. Y. $\mathrm{Li}^{3}$, J. K. Kim ${ }^{1}$, J. G. $\mathrm{Ju}^{1}$ and M. K. Song ${ }^{1} *$ \\ ${ }^{1}$ Department of Animal Science, Chungbuk National University, Cheongju, Chungbuk, Korea
}

\begin{abstract}
An in vitro experiment was conducted to examine the effects of defaunation (removal of protozoa) on ruminal fermentation characteristics, $\mathrm{CH}_{4}$ production and degradation by rumen microbes when incubated with cereal grains (corn, wheat and rye). Sodium lauryl sulfate as a defaunation reagent was added into the culture solution at a concentration of $0.000375 \mathrm{~g} / \mathrm{ml}$, and incubated anaerobically for up to $12 \mathrm{~h}$ at $39^{\circ} \mathrm{C}$. Following defaunation, live protozoa in the culture solution were rarely observed by microscopic examination. A difference in $\mathrm{pH}$ was found among grains regardless of defaunation at all incubation times ( $\mathrm{p}<0.01$ to $0.001)$. Defaunation significantly decreased $\mathrm{pH}$ at $12 \mathrm{~h}(\mathrm{p}<0.05)$ when rumen fluid was incubated with grains. Ammonia-N concentration was increased by defaunation for all grains at $6 \mathrm{~h}(\mathrm{p}<0.05)$ and $12 \mathrm{~h}(\mathrm{p}<0.05)$ incubation times. Total VFA concentration was increased by defaunation at $6 \mathrm{~h}(\mathrm{p}<0.05)$ and $12 \mathrm{~h}(\mathrm{p}<0.01)$ for all grains. Meanwhile, defaunation decreased acetate and butyrate proportions at $6 \mathrm{~h}(\mathrm{p}<0.05, \mathrm{p}<0.01)$ and $12 \mathrm{~h}(\mathrm{p}<0.01, \mathrm{p}<0.001)$, but increased the propionate proportion at $3 \mathrm{~h}, 6 \mathrm{~h}$ and $12 \mathrm{~h}$ incubation $(\mathrm{p}<0.01$ to 0.001$)$ for all grains. Defaunation increased in vitro effective degradability of $\mathrm{DM}(\mathrm{p}<0.05)$. Production of total gas and $\mathrm{CO}_{2}$ was decreased by defaunation for all grains at $1 \mathrm{~h}(\mathrm{p}<0.05, \mathrm{p}<0.05)$ and then increased at $6 \mathrm{~h}(\mathrm{p}<0.05, \mathrm{p}<0.05)$ and $12 \mathrm{~h}(\mathrm{p}<0.05$, $\mathrm{p}<0.05) . \mathrm{CH}_{4}$ production was higher from faunation than from defaunation at all incubation times $(\mathrm{p}<0.05)$. (Key Words: Defaunation, Grains, Fermentation, Degradation, Total Gas, $\mathrm{CH}_{4}$ )
\end{abstract}

\section{INTRODUCTION}

Cereal grains as the principal source of energy in the diets are widely used for intensive production of ruminant livestock all over the world. The primary component in grain is starch, which constitutes approximately 60 to $80 \%$ of the total ingredients of cereal grains (Huntington, 1997). Numerous studies (Ørskov, 1986; Huntington et al., 2006) have reported that the rumen is the main site of starch digestion, where the ruminal microorganisms contribute to 60 to $90 \%$ of the starch digestion. The rate and extent of starch digestion is also determined by processing approaches and the properties of starch granule (McAllister and Cheng, 1996). Thus, the potential cereal grain digestion is closely associated with the complex resident microflora

\footnotetext{
* Corresponding Author: Man K. Song. Tel: +82-43-261-2545, Fax: +82-43-273-2240, E-mail: mksong@cbnu.ac.kr

${ }^{2}$ Institute of Animal Science, Yanbian academy of agricultural sciences, Longjing, Jilin, China.

${ }^{3}$ Department of Animal Scicence, Yanbian University, Yanji, Jilin, China.
}

Submitted Apr. 30, 2012; Accepted Jun. 16, 2012; Revised Jul. 1, 2012 involved in the digestion process. Additionally, due to their numerical predominance and metabolic diversity, the ruminal bacteria have a key role in starch digestion in the rumen (Cheng et al., 1991). Ruminal protozoa are also believed to participate in the process by ingesting and digesting starch particles (Huntington, 1997). Some researchers (Coleman, 1992; Nagaraja et al., 1992) suggested that the presence of protozoa in the rumen can help ruminants fed high grain diets stabilize rumen fermentation through slowing starch digestion, thus reducing the risk of acidosis.

However, the fermentation of cereal grains in the rumen is generally accompanied by inevitable losses in heat and methane (Huntgate, 1966). It has been proven that protozoa are indirectly involved in methane production due to their close symbiotic relationship with methanogens, which allowed interspecies hydrogen transfer between them (Finlay et al., 1994). Based on this viewpoint, there is wide interest in investigating the effect of the elimination of protozoa (defaunation) on methane production. Some in vitro (Newbold et al., 1995) and in vivo (Morgavi et al., 2008) studies have also indicated that methanogens 
associated with protozoa contributed to 9 to $25 \%$ of methane production in the rumen. Meanwhile, Johnson and Johnson (1995) indicated that the energy loss through methane emission to the atmosphere represents 2 to $12 \%$ of the gross energy ingested by ruminants. Therefore, inhibition of methane production in the rumen can benefit not only ruminant production but also the environment (Becker and Wikselaar, 2011). Consequently, research has been focused on manipulating the number of protozoa to inhibit methane production (Schönhusen et al., 2003; Mohammed et al., 2004).

Many studies (Ørskov, 1986; Offner et al., 2003) are available describing the characteristics of starch degradation of cereal grains in the rumen. But little information is available on the relationship between cereal grain degradation and methane production as influenced by protozoa. A more efficient use of energy in ruminant diets could result from information regarding cereal grain metabolism and methane production in the rumen. The aim of this in vitro study, therefore, was to investigate the effects of defaunation of rumen fluid on fermentation, degradation and methane production when incubated with starch rich grain feeds.

\section{MATERIALS AND METHODS}

\section{Preparation of culture solution and its incubation}

Rumen contents were obtained $2 \mathrm{~h}$ after the morning feeding (09:00) from three ruminally-cannulated Holstein cows fed $9 \mathrm{~kg} / \mathrm{d}$ total diet daily $(7 \mathrm{~kg}$ concentrate and $2 \mathrm{~kg}$ ryegrass, as fed basis), feeding was done twice (09:00 and 18:00 h) daily, in an equal quantity. The rumen fluid was strained through 12 layers of cheesecloth to remove the feed particles. Carbon dioxide $\left(\mathrm{CO}_{2}\right)$ was flushed into the strained rumen fluid for $30 \mathrm{~s}$. Culture solution was prepared by mixing $40 \mathrm{ml}$ strained rumen fluid with $40 \mathrm{ml}$ McDougall's artificial saliva. The preparation of the defaunation of the culture solution was done according to the report of Abel et al. (2006). Sodium lauryl sulfate (Sigma, L5750) was added at a concentration of 0.000375 $\mathrm{g} / \mathrm{ml}$ as a defaunation reagent into the mixed culture solution to remove ruminal protozoa. The experiment was conducted essentially in a 2 (faunation and defaunation) $\times 3$ (three kinds of cereal grains) factorial design. Based on an air dried basis the nitrogen free extract (NFE) contents, as determined by proximal analyses using the AOAC (1995) method, were similar for $0.9 \mathrm{~g}$ corn, $1 \mathrm{~g}$ wheat and $1.1 \mathrm{~g}$ rye. These grains were ground through a $1 \mathrm{~mm}$ screen (Wiley mill) and were added to the culture solution with all microbes (faunation) or without protozoa (defaunation) in order to supply a similar amount of NFE contents. Degradability of the ground cereal grains was examined by preparing them in a small nylon bags $(5 \times 5 \mathrm{~cm}$, with pore
Table 1. Chemical composition of cereal grains added to the culture solutions (\%, DM basis)

\begin{tabular}{lcccc}
\hline Cereal & \multicolumn{4}{c}{ Chemical composition (\%, DM basis) } \\
\cline { 2 - 5 } grains & CP & EE & NDF & Ash \\
\hline Rye & 11.73 & 5.16 & 21.41 & 2.23 \\
Wheat & 9.57 & 4.89 & 18.85 & 1.48 \\
Corn & 7.08 & 3.04 & 16.05 & 1.20 \\
\hline
\end{tabular}

size of $50 \mu \mathrm{m}$ ) and placing them in $160 \mathrm{ml}$ bottles containing the mixed culture solution. The bottles were then sealed with rubber stoppers and were incubated anaerobically in a shaking incubator (VS-8480SR, VISON Science, Bucheon, Korea) at a speed of $135 \mathrm{rpm} / \mathrm{min}$ up to $12 \mathrm{~h}$ at $39^{\circ} \mathrm{C}$. The in vitro incubation was done 3 times in duplicate each time under similar conditions. The chemical composition of cereal grains added to the culture solution is shown in Table 1.

\section{Measurement and analysis}

Incubation was stopped by removing the bottles from the shaking incubator at $1,3,6$ and $12 \mathrm{~h}$, and $\mathrm{pH}$ of the culture solution was immediately measured. At the same time an aliquot of culture solution $(0.8 \mathrm{ml})$ was collected from each bottle for ammonia and volatile fatty acid (VFA) analysis. Ammonia-N concentration was determined by the method of Fawcett and Scott (1960) using a spectrophotometer. The $0.8 \mathrm{ml}$ of culture solution was mixed with $0.2 \mathrm{ml} 25 \%$ phosphoric acid and $0.2 \mathrm{ml}$ pivalic acid solution as the internal standard for the VFA analysis as described by $\mathrm{Li}$ et al. (2010). Total gas production was also measured at 1, 3, 6 and $12 \mathrm{~h}$ from the culture bottles through the 3-way stopcock connected to bottle using a 50 $\mathrm{ml}$ glass syringe. A gas sample was transferred to a $5 \mathrm{ml}$ vacuum tube and analyzed for methane $\left(\mathrm{CH}_{4}\right)$ and carbon dioxide $\left(\mathrm{CO}_{2}\right)$ by gas chromatograph (YL 6100GC, Young Lin Instrument Co., Korea) equipped with flame ionization detector (FID) and thermal conductivity detector (TCD). A $30 \mathrm{~m}$ silica capillary column (Agilent HP-PLQT Q, 19095P-Q04, $0.54 \mathrm{~mm}$ i.d., USA) was used to identify $\mathrm{CH}_{4}$ and $\mathrm{CO}_{2}$ peak analysis. The oven and injector temperatures for gas analysis were $100^{\circ} \mathrm{C}$ and $150^{\circ} \mathrm{C}$, and temperatures for FID and TCD detector were respectively kept at $230^{\circ} \mathrm{C}$ and $150^{\circ} \mathrm{C}$. The Nitrogen $\left(\mathrm{N}_{2}\right)$ gas was used as the carrier gas at a flow rate of $30 \mathrm{ml} / \mathrm{min}$. Nylon bags containing feed prior to and post incubation were washed with tap water and dried at $60^{\circ} \mathrm{C}$ for $48 \mathrm{~h}$ in a drying oven to measure dry matter (DM) degradation. Crude protein (CP), ether extract (EE), and organic acid (OM) were analyzed according to AOAC (1995). The neutral detergent fiber (NDF) was analyzed by the methods of Van Soest et al. (1991).

\section{Estimation of effective degradability in vitro}

Percent disappearance of DM at each incubation time 
was calculated from the portion remaining in the nylon bags after incubation. Disappearance rate was fitted to the equation of Ørskov and McDonald (1979):

$$
Y_{(\mathrm{t})}=\mathrm{a}+\mathrm{b}\left(1-\mathrm{e}^{-\mathrm{ct}}\right)
$$

Where $Y_{(\mathrm{t})}$ is the proportion of the incubated material degraded at time $t$; ' $a$ ' is the water soluble and instantly degradable fraction; ' $b$ ' is the potentially degradable fraction; ' $c$ ' is the fractional rate of degradation of fraction $b$ (h-1). Non linear parameters $\mathrm{a}, \mathrm{b}$ and $\mathrm{c}$ were estimated by an iterative least square procedure to calculate effective degradability of DM (EDDM) according to the following equation (Ørskov and McDonald, 1979):

\section{Effective degradability $=a+(b \times c) /(c+r)$}

Where ' $r$ ' is the fractional outflow rate and a hypothetical fractional outflow rate $(k \mathrm{p})$ of $0.05 \mathrm{~h}$ was used for estimation of effective degradability.

\section{Statiatical analyses}

Data were analyzed using the general linear models (GLM) procedure of SAS (V 9.1, 2002). Six treatments were replicated twice per time and repeated 3 times. For each variable measured at each time, replicates were averaged, and the total number of observations was 6 (treatments) $\times 3$ (times) $=18$ observations. The 18 observations obtained were subjected to least squares analysis of variance according to the following models:

$$
Y_{i j k}=\mu+\tau_{i}+S_{j}+O_{k}+\varepsilon_{i j k}
$$

Where $Y_{i j k}$ is dependent variable, $\mu$ is the overall mean, $\tau_{\mathrm{i}}$ is the fixed effect of treatment $(i=6), S_{j}$ is the random effect of repeated time $(j=3), O_{k}$ is the $j$ th incubation time and $\varepsilon_{i j k}$ is the error term.

Differences among treatments was considered, and the differences between faunation and defaunation was evaluated by pairwise t-test.

\section{RESULTS}

At each incubation time, microscopic examination was carried out to observe live protozoa through a 16/0.35 objective, and protozoa were rarely observed after $1 \mathrm{~h}$ incubation, indicating that live protozoa in the culture solution were almost eliminated by adding sodium lauryl sulfate. The $\mathrm{pH}$ of the culture solution decreased in all treatments regardless of defaunation as the incubation time advanced (Table 2). Defaunation significantly decreased ( $<<0.01$ to 0.001$) \mathrm{pH}$ of the culture solution for all of the grains except $1 \mathrm{~h}$ incubation compared with faunation.
Differences in $\mathrm{pH}$ of the culture solution was found between the grains regardless of defaunation $(\mathrm{p}<0.01$ to $<0.001)$. $\mathrm{pH}$ of culture solution from wheat and rye was relatively lower $(\mathrm{p}<0.001)$ than that from corn in both faunation and defaunation at $6 \mathrm{~h}$ and $12 \mathrm{~h}$ incubations. While $\mathrm{pH}$ from corn by faunation was the highest $(\mathrm{p}<0.001)$, $\mathrm{pH}$ from rye by defaunation was the lowest $(\mathrm{p}<0.001)$ after $12 \mathrm{~h}$ incubation.

Ammonia-N concentration of the culture solution increased for all the grains in both faunation and defaunation with advancing incubation times (Table 2). Defaunation increased ammonia-N concentration at $6 \mathrm{~h}$ $(\mathrm{p}<0.05)$ and $12 \mathrm{~h}(\mathrm{p}<0.05)$ incubation times compared with faunation. Ammonia-N concentration in the rye culture was the highest $(p<0.001)$ among the grains and then followed by wheat and corn.

Defaunation increased total VFA concentration at $6 \mathrm{~h}$ $(\mathrm{p}<0.05)$ and $12 \mathrm{~h}(\mathrm{p}<0.01)$ incubation time (Table 2) compared with faunation. The total VFA concentration from both faunation and defaunation also increased when incubated with wheat and rye at $6 \mathrm{~h}(\mathrm{p}<0.001)$ and $12 \mathrm{~h}$ $(p<0.001)$ incubation compared with corresponding values from corn. Furthermore, rye produced more total VFA $(\mathrm{p}<0.001)$ than wheat and corn at $6 \mathrm{~h}$ and $12 \mathrm{~h}$ incubation. Defaunation decreased proportions of acetate $\left(\mathrm{C}_{2}\right)$ at $6 \mathrm{~h}$ $(\mathrm{p}<0.05)$ and $12 \mathrm{~h}(\mathrm{p}<0.01)$ and butyrate $\left(\mathrm{C}_{4}\right)$ at $3 \mathrm{~h}$ $(\mathrm{p}<0.05), 6 \mathrm{~h}(\mathrm{p}<0.01)$ and $12 \mathrm{~h}(\mathrm{p}<0.01)$. Meanwhile, $\mathrm{C}_{2}$ proportion of corn from faunation and defaunation was higher than wheat and rye at $12 \mathrm{~h}(\mathrm{p}<0.001)$ incubation. Defaunation, however, increased $\mathrm{C}_{3}$ proportion for all cereal grains in comparison with faunation from $3 \mathrm{~h}$ incubation ( $\mathrm{p}<0.01$ to 0.001 ). Thus, defaunation decreased $\mathrm{C}_{2}$ to $\mathrm{C}_{3}$ ratio for all the grains from $3 \mathrm{~h}$ incubation $(\mathrm{p}<0.05$ to 0.001 ).

The effect of defaunation for grains on gas production is shown in Table 4. Defaunation firstly decreased total gas production at $1 \mathrm{~h}(\mathrm{p}<0.05)$ and $3 \mathrm{~h}(\mathrm{p}<0.05)$ and $\mathrm{CO}_{2}$ production at $1 \mathrm{~h}(\mathrm{p}<0.05)$ incubation, but then increased $(\mathrm{p}<0.001)$ total gas and $\mathrm{CO}_{2}$ production at $6 \mathrm{~h}(\mathrm{p}<0.05$, $\mathrm{p}<0.05)$ and $12 \mathrm{~h}(\mathrm{p}<0.05, \mathrm{p}<0.05)$ incubation for all grains. Rye in both faunation and defaunation cultures produced the highest amounts of total gas and $\mathrm{CO}_{2}$ through all incubation times $(\mathrm{p}<0.001)$ among grains in the present study. Defaunation clearly decreased $(\mathrm{p}<0.05) \quad \mathrm{CH}_{4}$ production at all the incubation times compared with faunation. However, corn resulted in a relatively lower $(p<0.001) \quad \mathrm{CH}_{4}$ generation from both faunation or defaunation than wheat and rye during $12 \mathrm{~h}$ incubations. In addition, defaunation was associated with a higher percent of $\mathrm{CO}_{2}(\mathrm{p}<0.05$ to 0.001$)$ and a lower percent of $\mathrm{CH}_{4}$ $(p<0.01$ to 0.001$)$ in total gas than faunation through all the incubation times. Similarly, defaunation resulted in lower ratio of $\mathrm{CH}_{4}$ to $\mathrm{CO}_{2}$ plus $\mathrm{CH}_{4}$ (p<0.01 to 0.001 ) and $\mathrm{CH}_{4}$ to $\mathrm{CO}_{2}(\mathrm{p}<0.01$ to 0.001$)$ than faunation. 
Table 2. $\mathrm{pH}$, ammonia-N concentration and concentration and proportions of major VFAs in the culture solution as influenced by faunation or defaunation when incubated with different cereal grains

\begin{tabular}{|c|c|c|c|c|c|c|c|c|c|}
\hline \multirow{3}{*}{ Items } & \multicolumn{6}{|c|}{ Treatment } & \multirow{3}{*}{ SEM $^{1}$} & \multirow{3}{*}{$\operatorname{Pr}>F^{2}$} & \multirow{3}{*}{$\begin{array}{l}\operatorname{Pr}>|t|^{3} \\
\text { F vs D }\end{array}$} \\
\hline & \multicolumn{3}{|c|}{ Faunation } & \multicolumn{3}{|c|}{ Defaunation } & & & \\
\hline & Corn & Wheat & Rye & Corn & Wheat & Rye & & & \\
\hline & & & & --------- & -------- & 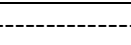 & & & \\
\hline $\mathrm{pH}$ & $7.47^{\mathrm{a}}$ & $7.35^{\mathrm{b}}$ & $7.26^{\mathrm{c}}$ & $7.47^{\mathrm{a}}$ & $7.45^{\mathrm{a}}$ & $7.39^{\mathrm{ab}}$ & 0.022 & $* * *$ & NS \\
\hline Ammonia (mg/100 ml) & $12.41^{\mathrm{c}}$ & $12.76^{\mathrm{c}}$ & $14.28^{\mathrm{ab}}$ & $13.46^{\mathrm{bc}}$ & $14.72^{\mathrm{a}}$ & $14.82^{\mathrm{a}}$ & 0.576 & $*$ & NS \\
\hline Total VFAs(mmoles/100 ml) & $55.04^{\mathrm{c}}$ & $62.15^{\mathrm{ab}}$ & $64.16^{\mathrm{a}}$ & $56.28^{\mathrm{bc}}$ & $56.78^{\mathrm{bc}}$ & $61.41^{\mathrm{ab}}$ & 1.450 & $* *$ & NS \\
\hline \multicolumn{10}{|c|}{ Molar proportion (mmoles/100 mmoles) } \\
\hline Acetate $\left(\mathrm{C}_{2}\right)$ & 67.59 & 68.17 & 66.75 & 67.18 & 67.60 & 67.90 & 0.457 & NS & NS \\
\hline Propionate $\left(\mathrm{C}_{3}\right)$ & 17.81 & 17.90 & 18.61 & 18.55 & 18.29 & 18.58 & 0.476 & NS & NS \\
\hline Butyrate $\left(\mathrm{C}_{4}\right)$ & 11.17 & 10.78 & 11.09 & 10.60 & 10.82 & 10.36 & 0.347 & NS & NS \\
\hline $\mathrm{C}_{2} / \mathrm{C}_{3}$ & 3.80 & 3.81 & 3.59 & 3.63 & 3.70 & 3.66 & 0.111 & NS & NS \\
\hline $\mathrm{pH}$ & $7.09^{\mathrm{a}}$ & $6.93^{\mathrm{ab}}$ & $6.84^{\mathrm{b}}$ & $7.00^{\mathrm{ab}}$ & $6.96^{\mathrm{ab}}$ & $6.84^{\mathrm{b}}$ & 0.039 & $* *$ & NS \\
\hline Ammonia (mg/100 ml) & $11.85^{\mathrm{b}}$ & $14.73^{\mathrm{a}}$ & $15.39^{\mathrm{a}}$ & $16.16^{\mathrm{a}}$ & $16.92^{\mathrm{a}}$ & $17.52^{\mathrm{a}}$ & 0.874 & $* *$ & NS \\
\hline Total VFAs (mmoles/100 ml) & 67.84 & 69.86 & 71.66 & 64.29 & 68.48 & 70.75 & 3.027 & NS & NS \\
\hline \multicolumn{10}{|c|}{ Molar proportion (mmoles/100mmoles) } \\
\hline Acetate $\left(\mathrm{C}_{2}\right)$ & 68.46 & 67.38 & 66.70 & 66.8 & 66.80 & 67.13 & 0.593 & NS & NS \\
\hline Propionate $\left(\mathrm{C}_{3}\right)$ & $17.08^{\mathrm{d}}$ & $17.74^{\mathrm{cd}}$ & $18.16^{\text {bcd }}$ & $19.91^{\mathrm{abc}}$ & $20.38^{\mathrm{ab}}$ & $20.80^{\mathrm{a}}$ & 0.617 & $* *$ & $* * *$ \\
\hline Butyrate $\left(\mathrm{C}_{4}\right)$ & $11.33^{\mathrm{a}}$ & $11.29^{\mathrm{a}}$ & $11.68^{\mathrm{a}}$ & $9.35^{\mathrm{b}}$ & $9.18^{\mathrm{b}}$ & $8.37^{\mathrm{b}}$ & 0.394 & $* * *$ & $*$ \\
\hline $\mathrm{C}_{2} / \mathrm{C}_{3}$ & $4.01^{\mathrm{a}}$ & $3.80^{\mathrm{ab}}$ & $3.67^{\mathrm{ab}}$ & $3.37^{\mathrm{b}}$ & $3.29^{\mathrm{b}}$ & $3.24^{\mathrm{b}}$ & 0.130 & $* *$ & $*$ \\
\hline $\mathrm{pH}$ & $6.83^{\mathrm{a}}$ & $6.70^{\mathrm{b}}$ & $6.49^{\mathrm{c}}$ & $6.62^{\mathrm{b}}$ & $6.19^{\mathrm{d}}$ & $6.09^{\mathrm{e}}$ & 0.029 & $* * *$ & NS \\
\hline Ammonia (mg/100 ml) & $12.11^{\mathrm{c}}$ & $14.79^{\mathrm{bc}}$ & $15.89^{\mathrm{ab}}$ & $18.26^{\mathrm{a}}$ & $18.88^{\mathrm{a}}$ & $19.55^{\mathrm{a}}$ & 0.879 & $* * *$ & $*$ \\
\hline Total VFAs (mmoles/100 ml) & $81.07^{\mathrm{d}}$ & $85.24^{\mathrm{cd}}$ & $96.18^{\mathrm{ab}}$ & $90.59^{\mathrm{bc}}$ & $99.98^{\mathrm{a}}$ & $103.21^{\mathrm{a}}$ & 2.287 & $* * *$ & $*$ \\
\hline \multicolumn{10}{|c|}{ Molar proportion (mmoles/100 mmoles) } \\
\hline Acetate $\left(\mathrm{C}_{2}\right)$ & $67.54^{\mathrm{a}}$ & $66.73^{\mathrm{ab}}$ & $66.02^{\mathrm{b}}$ & $61.76^{\mathrm{d}}$ & $62.52^{\mathrm{cd}}$ & $63.54^{\mathrm{c}}$ & 0.365 & $* * *$ & $*$ \\
\hline Propionate $\left(\mathrm{C}_{3}\right)$ & $17.35^{\mathrm{b}}$ & $18.07^{\mathrm{b}}$ & $18.58^{\mathrm{b}}$ & $27.29^{\mathrm{a}}$ & $26.75^{\mathrm{a}}$ & $26.95^{\mathrm{a}}$ & 0.652 & $* * *$ & $* *$ \\
\hline Butyrate $\left(\mathrm{C}_{4}\right)$ & $12.34^{\mathrm{a}}$ & $12.44^{\mathrm{a}}$ & $12.20^{\mathrm{a}}$ & $7.52^{\mathrm{b}}$ & $7.32^{\mathrm{b}}$ & $6.67^{\mathrm{b}}$ & 0.480 & $* * *$ & $* *$ \\
\hline $\mathrm{C}_{2} / \mathrm{C}_{3}$ & $3.90^{\mathrm{a}}$ & $3.71^{\mathrm{a}}$ & $3.56^{\mathrm{a}}$ & $2.26^{\mathrm{b}}$ & $2.34^{\mathrm{b}}$ & $2.36^{\mathrm{b}}$ & 0.116 & $* * *$ & $* *$ \\
\hline $\mathrm{pH}$ & $6.48^{\mathrm{a}}$ & $6.33^{\mathrm{ab}}$ & $6.38^{\mathrm{b}}$ & $5.96^{\mathrm{c}}$ & $5.68^{\mathrm{d}}$ & $5.63^{\mathrm{d}}$ & 0.034 & $* * *$ & $*$ \\
\hline Ammonia (mg/100 ml) & $14.11^{\mathrm{c}}$ & $17.47^{\mathrm{b}}$ & $19.43^{\mathrm{a}}$ & $17.63^{\mathrm{b}}$ & $20.22^{\mathrm{ab}}$ & $21.35^{\mathrm{ab}}$ & 0.759 & $* * *$ & $*$ \\
\hline Total VFAs (mmoles/100 ml) & $104.84^{\mathrm{e}}$ & $115.81^{\mathrm{d}}$ & $114.14^{\mathrm{d}}$ & $140.40^{\mathrm{c}}$ & $147.53^{\mathrm{b}}$ & $157.06^{\mathrm{a}}$ & 2.084 & $* * *$ & $* *$ \\
\hline \multicolumn{10}{|c|}{ Molar proportion (mmoles/100 mmoles) } \\
\hline Acetate $\left(\mathrm{C}_{2}\right)$ & $64.79^{\mathrm{a}}$ & $63.45^{\mathrm{b}}$ & $62.87^{\mathrm{b}}$ & $59.07^{\mathrm{c}}$ & $56.42^{\mathrm{d}}$ & $56.69^{\mathrm{d}}$ & 0.352 & $* * *$ & $* *$ \\
\hline Propionate $\left(\mathrm{C}_{3}\right)$ & $18.37^{\mathrm{c}}$ & $18.86^{\mathrm{c}}$ & $19.14^{\mathrm{c}}$ & $33.09^{\mathrm{b}}$ & $34.92^{\mathrm{a}}$ & $35.51^{\mathrm{a}}$ & 0.408 & $* * *$ & $* * *$ \\
\hline Butyrate $\left(\mathrm{C}_{4}\right)$ & $14.11^{\mathrm{a}}$ & $14.62^{\mathrm{a}}$ & $14.79^{\mathrm{a}}$ & $5.38^{\mathrm{b}}$ & $6.04^{\mathrm{b}}$ & $5.27^{\mathrm{b}}$ & 0.441 & $* * *$ & $* * *$ \\
\hline $\mathrm{C}_{2} / \mathrm{C}_{3}$ & $3.54^{\mathrm{a}}$ & $3.36^{\mathrm{ab}}$ & $3.29^{\mathrm{b}}$ & $1.79^{\mathrm{c}}$ & $1.62^{\mathrm{c}}$ & $1.60^{\mathrm{c}}$ & 0.059 & $* * *$ & $* * *$ \\
\hline
\end{tabular}

${ }^{1} \mathrm{SEM}=$ Standard error of means. ${ }^{2} \mathrm{Pr}>\mathrm{F}=$ Probability level.

${ }^{a, b, c, c}$ Means in the same row with different superscripts differ regardless of defaunation.

${ }^{3} \operatorname{Pr}>|t|=$ Probability level; Comparison between faunation and defaunation treatment $(\mathrm{F}=$ Faunation; $\mathrm{D}=$ Defaunation $)$

$* \mathrm{p}<0.05 ; * * \mathrm{p}<0.01 ; * * * \mathrm{p}<0.001 ; \mathrm{NS}=$ Non significant.

Defaunation significantly increased EDDM of grains $(\mathrm{p}<0.05)$ despite no differences in degradation parameters of $a, b$ and $c$. Within grains, regardless of defaunation, wheat and rye showed a higher EDDM and parameters $c$ $(p<0.001)$ than corn as shown in Table 3.

\section{DISCUSSION}

The rate and extent of starch fermentation are influenced by the grain type, the method of cereal grain processing and rumen microbe species (Hale, 1973; Ørskov, 1986). Some studies have shown that removal of protozoa generally resulted in a higher cereal grain degradation (Mackie et al., 1978; Mendoza et al., 1993). In the present experiment, our results on degradability were similar to those of previous reports. An explanation for this is that protozoa can manipulate the ruminal cereal grain hydrolysis process by engulfing numbers of bacteria to slow ruminal fermentation rates (Kurihara et al., 1978) or by ingesting starch granules and decreasing the accessibility of these 
Table 3. Degradation parameters (a, b, and c) and effective degradability (ED) DM of the experimental diets in the culture solution as influenced by faunation or defaunation when incubated with different cereal grains

\begin{tabular}{|c|c|c|c|c|c|c|c|c|c|}
\hline \multirow{3}{*}{$\begin{array}{l}\text { Parameters }^{1} \\
\text { and EDDM }\end{array}$} & \multicolumn{6}{|c|}{ Treatment } & \multirow{3}{*}{ SEM $^{2}$} & \multirow{3}{*}{$\operatorname{Pr}>F^{3}$} & \multirow{3}{*}{$\begin{array}{l}\operatorname{Pr}>|t|^{4} \\
\text { F vs D }\end{array}$} \\
\hline & \multicolumn{3}{|c|}{ Faunation } & \multicolumn{3}{|c|}{ Defaunation } & & & \\
\hline & Corn & Wheat & Rye & Corn & Wheat & Rye & & & \\
\hline $\mathrm{a}$ & 3.05 & 2.288 & 1.636 & 1.756 & 3.51 & 2.83 & 0.450 & NS & NS \\
\hline b & 60.28 & 64.99 & 66.66 & 79.09 & 74.08 & 71.84 & 5.018 & NS & NS \\
\hline $\mathrm{c}$ & $0.096^{\mathrm{c}}$ & $0.596^{\mathrm{b}}$ & $0.862^{\mathrm{a}}$ & $0.084^{\mathrm{c}}$ & $0.365^{\mathrm{b}}$ & $0.596^{\mathrm{b}}$ & 0.070 & $* * *$ & NS \\
\hline EDDM & $40.52^{\mathrm{d}}$ & $62.14^{\mathrm{b}}$ & $64.37^{\mathrm{ab}}$ & $49.76^{c}$ & $68.66^{\mathrm{a}}$ & $69.11^{\mathrm{a}}$ & 1.321 & $* * *$ & $*$ \\
\hline
\end{tabular}

${ }^{1} \mathrm{a}=$ Intercept representing rapidly soluble fraction in the rumen; $\mathrm{b}=$ Fraction of degradable at time infinity; $\mathrm{c}=$ Rate constant of disappearance of fraction "b".

${ }^{2} \mathrm{SEM}=$ Standard error of means. ${ }^{3} \mathrm{Pr}>\mathrm{F}=$ Probability level. ${ }^{\text {a,b.c }}$ Means in the same row with different superscripts differ regardless of defaunation.

${ }^{4} \operatorname{Pr}>|\mathrm{t}|=$ Probability level; Comparison between faunation and defaunation treatment $(\mathrm{F}=$ Faunation; $\mathrm{D}=\mathrm{Defaunation})$.

$* \mathrm{p}<0.05 ; * * \mathrm{p}<0.01 ; * * * \mathrm{p}<0.001 ; \mathrm{NS}=$ Non significant.

Table 4. Gas production in the culture solution as influenced by faunation or defaunation when incubated with different cereal grains

\begin{tabular}{|c|c|c|c|c|c|c|c|c|c|}
\hline \multirow{3}{*}{ Items } & \multicolumn{6}{|c|}{ Treatment } & \multirow{3}{*}{ SEM $^{1}$} & \multirow{3}{*}{$\operatorname{Pr}>F^{2}$} & \multirow{3}{*}{$\begin{array}{l}\operatorname{Pr}>|t|^{3} \\
\text { F vs D }\end{array}$} \\
\hline & \multicolumn{3}{|c|}{ Faunation } & \multicolumn{3}{|c|}{ Defaunation } & & & \\
\hline & Corn & Wheat & Rye & Corn & Wheat & Rye & & & \\
\hline & ---------- & 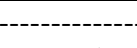 & -------- & h ---------- & --------- & ---------- & & & \\
\hline Total gas (ml) & $14.83^{\mathrm{c}}$ & $17.33^{\mathrm{b}}$ & $21.50^{\mathrm{a}}$ & $9.92^{\mathrm{d}}$ & $10.75^{\mathrm{d}}$ & $11.67^{\mathrm{d}}$ & 0.563 & $* * *$ & $*$ \\
\hline $\mathrm{CO}_{2}(\mathrm{ml})$ & $10.78^{\mathrm{c}}$ & $12.45^{\mathrm{b}}$ & $15.65^{\mathrm{a}}$ & $7.96^{\mathrm{e}}$ & $8.75^{\mathrm{de}}$ & $9.73^{\mathrm{cd}}$ & 0.460 & $* * *$ & $*$ \\
\hline $\mathrm{CH}_{4}(\mathrm{ml})$ & $3.47^{\mathrm{c}}$ & $4.24^{\mathrm{b}}$ & $5.19^{\mathrm{a}}$ & $1.43^{\mathrm{d}}$ & $1.57^{\mathrm{d}}$ & $1.69^{\mathrm{d}}$ & 0.106 & $* * *$ & $*$ \\
\hline $\mathrm{CO}_{2} \%$ in total gas & $72.65^{\mathrm{b}}$ & $71.83^{\mathrm{b}}$ & $72.79^{b}$ & $80.45^{\mathrm{a}}$ & $81.52^{\mathrm{a}}$ & $83.54^{\mathrm{a}}$ & 1.601 & $* *$ & $* *$ \\
\hline $\mathrm{CH}_{4} \%$ in total gas & $23.40^{\mathrm{a}}$ & $24.50^{\mathrm{a}}$ & $24.14^{\mathrm{a}}$ & $14.45^{\mathrm{b}}$ & $15.79^{\mathrm{b}}$ & $13.39^{\mathrm{b}}$ & 0.644 & $* * *$ & $* *$ \\
\hline $\mathrm{CH}_{4} /\left(\mathrm{CH}_{4}+\mathrm{CO}_{2}\right)$ & $0.244^{\mathrm{a}}$ & $0.254^{\mathrm{a}}$ & $0.249^{\mathrm{a}}$ & $0.152^{\mathrm{b}}$ & $0.162^{\mathrm{b}}$ & $0.138^{\mathrm{b}}$ & 0.010 & $* * *$ & $* *$ \\
\hline $\mathrm{CH}_{4} / \mathrm{CO}_{2}$ & $0.323^{\mathrm{a}}$ & $0.341^{\mathrm{a}}$ & $0.332^{\mathrm{a}}$ & $0.180^{\mathrm{b}}$ & $0.194^{\mathrm{b}}$ & $0.161^{\mathrm{b}}$ & 0.006 & $* * *$ & $* *$ \\
\hline Total gas (ml) & $35.17^{\mathrm{c}}$ & $44.63^{\mathrm{b}}$ & $53.83^{\mathrm{a}}$ & $31.47^{\mathrm{c}}$ & $42.37^{\mathrm{b}}$ & $49.67^{\mathrm{a}}$ & 1.365 & $* * *$ & $*$ \\
\hline $\mathrm{CO}_{2}(\mathrm{ml})$ & $24.25^{\mathrm{d}}$ & $30.03^{c}$ & $36.52^{\mathrm{b}}$ & $25.83^{\mathrm{d}}$ & $34.29^{\mathrm{b}}$ & $40.16^{\mathrm{a}}$ & 0.990 & $* * *$ & NS \\
\hline $\mathrm{CH}_{4}(\mathrm{ml})$ & $8.81^{\mathrm{c}}$ & $11.10^{\mathrm{b}}$ & $13.54^{\mathrm{a}}$ & $4.91^{\mathrm{d}}$ & $6.66^{\mathrm{cd}}$ & $7.00 \mathrm{c}^{\mathrm{d}}$ & 0.635 & $* * *$ & $*$ \\
\hline $\mathrm{CO}_{2} \%$ in total gas & $69.00^{\mathrm{b}}$ & $67.87^{\mathrm{b}}$ & $67.79^{\mathrm{b}}$ & $82.08^{\mathrm{a}}$ & $81.00^{\mathrm{a}}$ & $80.93^{\mathrm{a}}$ & 1.838 & $* * *$ & $* * *$ \\
\hline $\mathrm{CH}_{4} \%$ in total gas & $24.97^{\mathrm{a}}$ & $25.06^{\mathrm{a}}$ & $25.11^{\mathrm{a}}$ & $15.60^{\mathrm{b}}$ & $15.68^{\mathrm{b}}$ & $14.08^{\mathrm{b}}$ & 1.298 & $* * *$ & $* *$ \\
\hline $\mathrm{CH}_{4} /\left(\mathrm{CH}_{4}+\mathrm{CO}_{2}\right)$ & $0.265^{\mathrm{a}}$ & $0.270^{\mathrm{a}}$ & $0.270^{\mathrm{a}}$ & $0.160^{\mathrm{b}}$ & $0.162^{\mathrm{b}}$ & $0.149^{\mathrm{b}}$ & 0.022 & $* * *$ & $* *$ \\
\hline $\mathrm{CH}_{4} / \mathrm{CO}_{2}$ & $0.365^{\mathrm{a}}$ & $0.370^{\mathrm{a}}$ & $0.371^{\mathrm{a}}$ & $0.190^{\mathrm{b}}$ & $0.194^{\mathrm{b}}$ & $0.174^{\mathrm{b}}$ & 0.012 & $* * *$ & $* *$ \\
\hline Total gas (ml) & $62.50^{f}$ & $80.03^{\mathrm{d}}$ & $90.50^{\mathrm{c}}$ & $72.14^{\mathrm{e}}$ & $101.64^{\mathrm{b}}$ & $112.00^{\mathrm{a}}$ & 1.324 & $* * *$ & $*$ \\
\hline $\mathrm{CO}_{2}(\mathrm{ml})$ & $41.42^{\mathrm{f}}$ & $53.13^{\mathrm{e}}$ & $64.33^{c}$ & $58.24^{\mathrm{d}}$ & $78.59^{\mathrm{b}}$ & $92.86^{\mathrm{a}}$ & 1.603 & $* * *$ & $*$ \\
\hline $\mathrm{CH}_{4}(\mathrm{ml})$ & $17.10^{\mathrm{c}}$ & $21.22^{\mathrm{b}}$ & $25.10^{\mathrm{a}}$ & $11.39^{\mathrm{d}}$ & $15.85^{\mathrm{c}}$ & $17.03^{\mathrm{c}}$ & 0.638 & $* * *$ & $*$ \\
\hline $\mathrm{CO}_{2} \%$ in total gas & $66.22^{\mathrm{c}}$ & $66.40^{\mathrm{c}}$ & $70.88^{\mathrm{c}}$ & $80.80^{\mathrm{ab}}$ & $77.29^{\mathrm{b}}$ & $82.90^{\mathrm{a}}$ & 1.440 & $* * *$ & $* *$ \\
\hline $\mathrm{CH}_{4} \%$ in total gas & $27.39^{\mathrm{a}}$ & $26.49^{\mathrm{a}}$ & $27.74^{\mathrm{a}}$ & $15.77^{\mathrm{b}}$ & $15.59^{\mathrm{b}}$ & $15.21^{\mathrm{b}}$ & 0.585 & $* * *$ & $* *$ \\
\hline $\mathrm{CH}_{4} /\left(\mathrm{CH}_{4}+\mathrm{CO}_{2}\right)$ & $0.293^{\mathrm{a}}$ & $0.285^{\mathrm{a}}$ & $0.281^{\mathrm{a}}$ & $0.163^{\mathrm{b}}$ & $0.168^{\mathrm{b}}$ & $0.155^{\mathrm{b}}$ & 0.011 & $* * *$ & $* * *$ \\
\hline $\mathrm{CH}_{4} / \mathrm{CO}_{2}$ & $0.414^{\mathrm{a}}$ & $0.399^{\mathrm{a}}$ & $0.391^{\mathrm{a}}$ & $0.195^{\mathrm{b}}$ & $0.202^{\mathrm{b}}$ & $0.183^{\mathrm{b}}$ & 0.006 & $* * *$ & $* * *$ \\
\hline Total gas (ml) & $109.50^{\mathrm{d}}$ & $128.36^{\mathrm{c}}$ & $147.17^{\mathrm{b}}$ & $125.81^{\mathrm{c}}$ & $151.69^{\mathrm{b}}$ & $163.33^{\mathrm{a}}$ & 1.959 & $* * *$ & $*$ \\
\hline $\mathrm{CO}_{2}(\mathrm{ml})$ & $77.75^{\mathrm{e}}$ & $87.93^{\mathrm{d}}$ & $100.03^{\mathrm{c}}$ & $101.95^{\mathrm{c}}$ & $123.69^{\mathrm{b}}$ & $134.45^{\mathrm{a}}$ & 2.344 & $* * *$ & $*$ \\
\hline $\mathrm{CH}_{4}(\mathrm{ml})$ & $29.53^{\mathrm{c}}$ & $34.82^{\mathrm{b}}$ & $38.68^{\mathrm{a}}$ & $19.88^{\mathrm{e}}$ & $23.15^{\mathrm{d}}$ & $23.72^{\mathrm{d}}$ & 0.922 & $* * *$ & $*$ \\
\hline $\mathrm{CO}_{2} \%$ in total gas & $71.01^{\mathrm{b}}$ & $68.47^{\mathrm{b}}$ & $67.96^{\mathrm{b}}$ & $81.01^{\mathrm{a}}$ & $81.54^{\mathrm{a}}$ & $82.32^{\mathrm{a}}$ & 0.953 & $* * *$ & * \\
\hline $\mathrm{CH}_{4} \%$ in total gas & $26.97^{\mathrm{a}}$ & $27.14^{\mathrm{a}}$ & $26.29^{\mathrm{a}}$ & $15.79^{\mathrm{b}}$ & $15.26^{\mathrm{b}}$ & $14.52^{\mathrm{b}}$ & 0.649 & $* * *$ & $* * *$ \\
\hline $\mathrm{CH}_{4} /\left(\mathrm{CH}_{4}+\mathrm{CO}_{2}\right)$ & $0.275^{\mathrm{a}}$ & $0.284^{\mathrm{a}}$ & $0.279^{\mathrm{a}}$ & $0.163^{\mathrm{b}}$ & $0.158^{\mathrm{b}}$ & $0.149^{\mathrm{b}}$ & 0.005 & $* * *$ & $* *$ \\
\hline $\mathrm{CH}_{4} / \mathrm{CO}_{2}$ & $0.380^{\mathrm{a}}$ & $0.397^{\mathrm{a}}$ & $0.387^{\mathrm{a}}$ & $0.195^{\mathrm{b}}$ & $0.187^{\mathrm{b}}$ & $0.175^{\mathrm{b}}$ & 0.10 & $* * *$ & $* *$ \\
\hline
\end{tabular}

\footnotetext{
${ }^{1} \mathrm{SEM}=$ Standard error of means. ${ }^{2} \mathrm{Pr}>\mathrm{F}=$ Probability level.

${ }_{\text {a,b.c }}$ Means in the same row with different superscripts differ regardless of defaunation.

${ }^{3} \operatorname{Pr}>|t|=$ Probability level; Comparison between faunation and defaunation treatment $(\mathrm{F}=$ Faunation; $\mathrm{D}=$ Defaunation $)$

$* \mathrm{p}<0.05 ; * * \mathrm{p}<0.01 ; * * * \mathrm{p}<0.001 ; \mathrm{NS}=$ Non significant.
} 
substrates to prevent its immediate and rapid fermentation by bacteria (Coleman, 1986; Coleman, 1992). Thus both of these factors can decrease degradation of cereal grains and our results indicate that the defaunation agent used in the present study showed strong toxicity to rumen protozoa but without effect on bacteria. This selective toxicity can be explained in that the defaunation agent reacts with cholesterol in eukaryotic membranes but not in prokaryotic cells (Wina et al., 2005), causing protozoal cells to rupture and lyse (Kilta et al., 1996). In addition, the three cereal grains used in the present experiment also differed significantly in EDDM regardless of the presence of protozoa. As expected, wheat and rye had relatively higher EDDM than corn, suggesting that wheat and rye starch was more rapidly fermented by ruminal microbes than corn. Similar results were also observed by others (Ørskov, 1986; McAllister et al., 1990; Lanzas et al., 2007). Decreased EDDM in corn might be simply due to thickness of the protein matrix which coats starch granules (McAllister and Cheng, 1996), and this matrix is relatively difficult to be hydrolyzed by water and enzymes (McAllister et al., 1990). On the other hand, the distribution of starch granules within the kernel varies with cereal grain type (Swan et al., 2006). The starch granules in rye and wheat endosperms seem to be floury and have a relatively small particle size. Consequently, the smaller starch granules have a larger surface area available for microbial and enzymatic starch hydrolysis which results in rapid degradation.

In the present study, defaunation resulted in lower $\mathrm{pH}$ compared with faunation, which is in agreement with other research (Nagaraja et al., 1992; Mendoza et al., 1993). The difference in ruminal $\mathrm{pH}$ between the faunated and defaunated cultures was related to total VFA concentration. Thus, the increase in total VFA concentration that was observed from defaunation could be responsible for the lower $\mathrm{pH}$.

Some studies (Abel et al., 2006; Kiran and Mutsvangwa, 2010) reported that defaunation generally led to a decrease in $\mathrm{NH}_{3}-\mathrm{H}$ concentration. However, our results in the current study are in contrast to the previous reports. One of the possible reasons for this is that the eliminated protozoa in the culture solution were a source of microbial protein and contributed to the additional $\mathrm{NH}_{3}-\mathrm{H}$ production.

The rate and extent of DM digestion may influence the proportion of VFA produced in culture solution. In the present in vitro study, defaunation firstly tended to slightly decrease the total VFA concentration at $1 \mathrm{~h}$ and $3 \mathrm{~h}$ incubations, and this may be related to the defaunating process. But after complete elimination of live protozoa from the culture solution, defaunation led to a significant increase in total VFA concentration up to $12 \mathrm{~h}$ incubation. Results of the present experiment are in line with some other reports (Nagaraja et al., 1992; Abel et al., 2006). The higher total VFA concentration might be closely related to the increased EDDM by defaunation. Increased total VFA concentration by defaunation might be due to increased bacterial numbers (Nagaraja et al., 1992). It generally has been observed that bacterial populations are larger after defaunation (Hristov et al., 2001), possibly reflecting both decreased engulfing of bacteria by protozoa and the killed protozoa supplying additional substrate for bacterial reproduction, thus resulting in a higher level of total VFA production (Williams and Withers, 1991; Williams and Coleman, 1992). Additionally, defaunation increased the molar proportion of $\mathrm{C} 3$ but decreased the molar proportions of $\mathrm{C} 2$ and $\mathrm{C} 4$. Numerous studies (Williams and Withers, 1991; Nagaraja et al., 1992; Eugene et al., 2004) have also reported that increased $\mathrm{C} 3$ was often accompanied by decreases in $\mathrm{C} 2$ and $\mathrm{C} 4$ proportions. The shift in proportions of VFA may be associated with the selective engulfment of starch-hydrolyzing bacteria by protozoa (Kurihara et al., 1968). It has been indicated that the high C3 proportion may result from increased bacterial numbers and a shift in the predominant bacterial species by defaunation. Thus, defaunation resulted in a lower $\mathrm{C} 2$ to $\mathrm{C} 3$ ratio compared with faunation. The individual cereal grains appeared with almost the same trend in VFA characteristics between faunation and defaunation. Meanwhile, rye showed similar results to wheat from the defaunation treatment in comparison with corn, which corresponded with its result of EDDM. That would indicate that NFE content in rye may be more rapidly available for rumen microbes, thus stimulating microbial growth and VFA production.

Our results showed a positive correlation for cereal grains between total gas production and EDDM. Chai et al. (2004) suggested that gas measurement can be considered as a method to estimate potential starch degradation. Thus, the increased total gas production which occurred from these cereal grains from both faunation and defaunation can be attributed to the higher EDDM in the culture solution. Furthermore, our results in the present study also showed a higher percent of $\mathrm{CO}_{2}$ and a lower percent of $\mathrm{CH}_{4}$ in the total gas from defaunated cultures. This indicated that the increase in total gas production might be accompanied by increased $\mathrm{CO}_{2}$ production rather than $\mathrm{CH}_{4}$. Metabolic $\mathrm{H}_{2}$ consumed by methanogens is the principal pathway for $\mathrm{CH}_{4}$ generation in the rumen and the quantity of methane generated is closely related to the end products of carbohydrate fermentation (Yane-Ruiz et al., 2010). Castillo et al. (2004) and Li et al. (2009 a, b) reported that increased C3 production resulted in suppressed methane production due to competition with methanogens for the available $\mathrm{H}_{2}$. These findings are in line with our results that lower methane production from defaunated cultures was accompanied by increased C3 production (Table 2). Additionally, protozoa also play an important role on 
methanogensis, which could be related to their participation in $\mathrm{H}_{2}$ metabolism or their influences on methanogen populations and the species of the microbiota. Based on the results of in vivo and in vitro trials Hegarty (1999) and Morgavi et al. (2010) summarized that the complete elimination of protozoa from the rumen would result in a $13 \%$ decrease in methane production. Defaunation significantly reduced $\mathrm{CH}_{4}$ production for all the grain feeds in the current study compared with faunation (Table 4). Finlay et al. (1994) described a symbiotic relationship between ciliate protozoa and methanogens that are found inside or in close association with protozoal cells, which has been established to allow an interspecies $\mathrm{H}_{2}$ transfer between ciliate protozoa and methanogens. This indicates that protozoa indirectly contributed to methane production. In the present study, defaunation decreased $\mathrm{CH}_{4}$ production by $11 \%$ compared with fauantion (Table 4 ). Therefore, the number and activity of methanogens are believed to be indirectly restricted by defaunation, and the disturbance of the synergy between protozoa and methanogens could be partly responsible for the reduction of $\mathrm{CH}_{4}$ production. Meanwhile, other similar defauantion experiments have proved that methanogens were not directly affected by adding a defaunation reagent (Dohme et al., 1999), thus indicating that the present defaunation agent could be applied to eliminate protozoa to study its effects on methanogenesis by methanogens. On the other hand, the difference in gas proportions was not found between cereal grains from both faunation and defaunation cultures. One of the possible reasons for these results might be due to the fact that we used the same amount of NFE content for all the cereal grains, thus resulting in a similar establishment of microbial groups.

Based on the results obtained from the present experiment, it is concluded that defaunation can widely modify the in vitro fermentation pattern resulting in a higher EDDM, shifts in molar proportion of VFA production and a reduction of methane emission. Modification of the fermentation pattern can be attributed to the change of rumen micro-flora caused by the elimination of protozoa. Besides, according to the metabolic characteristics of cereal grains in the present study, wheat and rye seem to be more sensitive to this modification when compared with corn. However, more detailed studies are needed to conduct to verify these findings.

\section{REFERENCES}

Abel, H., B. Schröder, P. Lebzien and G. Flachowsky. 2006. Effects of defaunation on fermentation characteristics and biotin balance in an artificial rumen-simulation system (RUSITEC) receiving diets with different amounts and types of cereal. Br. J. Nutr. 95:99-104.

AOAC. 1995. Official methods of analsis, 13th edn. Association of official analytical chemists, Washington, DC, USA

Becker, P. M. and P. G. Wikselaar. 2011. Effects of plant antioxidants and natural vicinal diketones on methane production, studied in vitro with rumen fluid and a polylactate as maintenance substrate. Anim. Feed Sci. Technol. 170:201208.

Castillo, C., J. Benedito, J. Mendez, V. Pereira, M. Lopez-Alonso, M. Miranda and J. Hernandez. 2004. Organic acids as a substitute for monensin in diets for beef cattle. Anim. Feed Sci. Technol. 115:101-116.

Chai, W. Z., A. H. Van Gelder and J. W. Cone. 2004. Relationship between gas production and starch degradation in feed samples. Anim. Feed Sci. Technol. 114:195-204.

Cheng, K. J., C. W. Forsberg, H. Minato and J. W. Costerton. 1991. Microbial ecology and physiology of feed degradation within the rumen. In: Physiological Aspects of Digestion and Metabolism in Ruminants (Ed. R. Kawashima). Academic Press, Toronto, Ont., pp. 595-624.

Coleman, G. S. 1986. The amylase activity of 14 species of entodiniomorphid protozoa and the distribution of amylase in rumen digesta fractions of sheep containing no protozoa or one of seven different protozoal populations. J. Agric. Sci. (Camb.) 107:709-721.

Coleman, G. S. 1992. The rate of uptake and metabolism of starch grains and cellulose particles by Entodinium species, Eudiplodinium maggi, some other entodinomorphid protozoa and natural protozoal populations taken from the ovine rumen. J. Appl. Microbiol. 73:507-513.

Dohme, F., A. Machmüller, B. L. Estermann, P. Pfister, A. Wasserfallen and M. Kreuzer. 1999. The role of the rumen ciliate protozoa for methane suppression caused by coconut oil. Lett. Appl. Microbiol. 29:187-192.

Eugene, M., H. Archimede and D. Sauvant. 2004. Quantitative meta-analysis on the effects of defaunation of the rumen on growth, intake and digestion in ruminants. Livest. Sci. 85:8197.

Fawcett, J. K. and J. E. Scott. 1960. A rapid and precise method for the determination of urea. J. Clin. Pathol. 13:156-163.

Finlay, B. J., G. Esteban, K. J. Clarke, A. G. Williams, T. M. Embley and R. R. Hirt. 1994. Some rumen ciliates have endosymbiotic methanogens. FEMS. Microbiol. Lett. 117: 157-162.

Hale, W. H. 1973. Influence of processing on the utilization of grains (starch) by ruminant. J. Anim. Sci. 37:1075-1080.

Hegarty, R. S. 1999. Reducing rumen methane emissions through elimination of rumen protozoa. Aust. J. Agric. Res. 50:13211327.

Hristov, A. N., M. Ivan, L. M. Rode and T. A. McAllister. 2001. Fermentation characteristics and ruminal ciliate protozoal populations in cattle fed medium-or high- concentrate barleybased diets. J. Anim. Sci. 79:515-524.

Hungate, R. E. 1966. The Rumen and its Microbes. Academic press, New York.

Huntington, G. B. 1997. Starch utilization by ruminants: From basics to the bunk. J. Anim. Sci. 75:852-867.

Huntington, G. B., D. L. Harmon and C. J. Richards. 2006. Site, rates, and limits of starch digestion and glucose metabolism in growing cattle. J. Anim. Sci. 84:14-24.

Johnson, K. A. and D. E. Johnson. 1995. Methane emissions from 
cattle. J. Anim. Sci. 73:2483-2492.

Kilta, P. T., G. W. Mathison and T. W. Fenton. 1996. Effect of alfalfa root saponins on digestive function in sheep. J. Anim. Sci. 74:1144-1156.

Kiran, D. and T. Mutsvangwa. 2010. Effects of partial ruminal defaunation on urea-nitrogen recycling, nitrogen metabolism, and microbial nitrogen supply in growing lambs fed low or high dietary crude protein concentrations. J. Anim. Sci. 88: 1034-1047.

Kurihara, Y., J. M. Eadie, P. N. Hobson and S. O. Mann. 1968. Relationship between bacteria and ciliate protozoa in the sheep rumen. J. Gen. Microbiol. 51:267-288.

Kurihara, Y., T. Takechi and F. Shibata. 1978. Relationship between bacteria and ciliate protozoa in the rumen of a sheep fed a purified diet. J. Agric. Sci. (Camb.) 90:373-382.

Lanzas, C., D. G. Fox and A. N. Pell. 2007. Digestion kinetics of dried cereal grains. Anim. Feed Sci. Technol. 136:265-280.

Li, X. Z., C. G. Yan, S. H. Choi, R. J. Long, G. L. Jin and M. K. Song. 2009a. Effects of addition level and chemical type of propionate precursors in dicarboxylic acid pathway on fermentation characteristics and methane production by rumen microbes in vitro. Asian-Aust. J. Anim. Sci. 22:82-89.

Li, X. Z., R. J. Long, C. G. Yan, S. H. Choi, G. L. Jin and M. K. Song. 2010. Rumen microbial responses in fermentation characteristics and production of CLA and methane to linoleic acid in associated with malate or fumarate. Anim. Feed Sci. Technol. 155:132-139.

Li, X. Z., S. H. Choi, G. L. Jin, C. G. Yan, R. J. Long, C. Y. Liang and M. K. Song. 2009b. Linolenic acid in association with malate or fumarate increased CLA production and reduced methane generation by rumen microbes. Asian-Aust. J. Anim. Sci. 22:819-826.

Mackie, R. I., F. M. C. Gilchrist, A. M. Roberts, P. E. Hannah and H. M. Schwartz. 1978. Microbiological and chemical changes in the rumen during the stepwise adaptation of sheep to high concentrate diets. J. Agric. Sci. (Camb.) 90:241-254.

McAllister, T. A. and K. J. Cheng. 1996. Microbial strategies in the ruminal digestion of cereal grains. Anim. Feed Sci. Technol. 62:29-36.

McAllister, T. A., L. M. Rode, D. J. Major, K. J. Cheng and J. G. Buchanan-Smith. 1990c. Effect of ruminal microbial colonization on cereal gram digestion. Can. J. Anim. Sci. 70: 571-579.

Mendoza, G. D., R. A. Britton and R. A. Stock. 1993. Influence of ruminal protozoa on site and extent of starch digestion and ruminal fermentation. J. Anim. Sci. 71:1572-1578.

Mohammed, N., N. Ajisaka, Z. A. Lila, K. Hara, K. Mikuni, K. Hara, S. Kanda and H. Itabashi. 2004. Effect of Japanese horseradish oil on methane production and ruminal fermentation in vitro and in steers. J. Anim. Sci. 82:1839-1846.
Morgavi, D. P., E. Forano, C. Martin and C. J. Newbold. 2010. Microbial ecosystem and methanogenesis in ruminants. Animal 4:1024-1036.

Morgavi, D. P., J. P. Jouany and C. Martin. 2008. Changes in methane emission and rumen fermentation parameters induced by refaunation in sheep. Aust. J. Exp. Agric. 48:69-72.

Nagaraja, T. G., G. Towne and A. A. Beharka. 1992. Moderation of ruminal fermentation by ciliated protozoa in cattle fed a highgrain diet. Appl. Environ. Microbiol. 58:2410-2414.

Newbold, C. J., B. Lassalas and J. P. Jouany. 1995. The importance of methanogens associated with ciliate protozoa in ruminal methane production in vitro. Lett. Appl. Microbiol. 27:230-234.

Offner, A., A. Bach and D. Sauvant. 2003. Quantitative review of in situ starch degradation in the rumen. Anim. Feed Sci. Technol. 106:81-93.

Ørskov, E. R. 1986. Starch digestion and utilization in ruminants. J. Anim. Sci. 63:1624-1633.

Ørskov, E. R. and I. McDonald. 1979. The estimation of protein degradability in the rumen from incubation measurements weighted according to rate of passage. J. Agric. Sci. (Camb.) 92:499-506.

SAS Inc. 2002. SAS User's guide: Statistical analysis system institute, SAS Inc., Cary, NC, USA.

Schönhusen, U., R. Zitnan, S. Kuhla, W. Jentsch, M. Derno and J. Voiqt. 2003. Effects of protozoa on methane production in rumen and hindgut of calves around time of weaning. Arch. Anim. Nutr. 57:279-295.

Steel, R. G. D. and J. H. Torrie. 1980. Principles and procedures of statistics. Mcgraw Hill Book Co., NY, USA.

Swan, C. G., J. G. P. Bowman, J. M. Martin and M. J. Giroux. 2006. Incresed puroindoline levels slow ruminal digestion of wheat (Triticum aestivum L.) starch by cattle. J. Anim. Sci. 84:641650

Van Soest, P. J., J. B.Robertson and B. A. Lewis. 1991. Methods for dietary fiber, neutral detergent fiber, and nonstarch polysaccharides in relation to animal nutrition. J. Dairy Sci. 74:3583-3597.

Williams, A. G. and G. S. Coleman. 1992. The Rumen Protozoa. Springer-Verlag, New York, USA.

Williams, A. G. and S. E.Withers. 1991. Effect of ciliate protozoa on the activity of polysaccharide-degrading enzymes and fibre breakdown in the rumen ecosystem. J. Appl. Microbiol.70: 144-155.

Wina, E., S. Muetzel and K. Becker. 2005. The impact of saponins or saponin containing plant materials on ruminant productionA Review. J. Agric. Food. Chem. 53:8093-8105.

Yanez-Ruiz, D. R., B. Macias, E. Pinloche and C. Newbold. 2010. The persistence of bacterial and methanogenic archaeal communities residing in the rumen of yong lambs. FEMS. Microbiol. Ecol. 72:272-278. 